\title{
Reward contrast in delay and probability discounting
}

\author{
ZhiJie Dai, Randolph C. Grace, ANd Simon Kemp \\ University of Canterbury, Christchurch, New Zealand
}

\begin{abstract}
In this study, we examined whether reward contrast influences choice between delayed and probabilistic outcomes. Specifically, we predicted that the subjective value of an intermediate reward would seem relatively larger or smaller, respectively, if it followed choices involving a smaller or larger reward and would produce corresponding changes in rates of delay and probability discounting. In Experiment 1, subjects made choices about hypothetical $\$ 5,000$ or $\$ 50$ outcomes and then made choices about $\$ 500$ outcomes. Delay-discounting rates for the $\$ 500$ outcome were larger for Group $\$ 5,000$ than for Group $\$ 50$, whereas the opposite result was obtained for probability-discounting rates. In Experiment 2, we used a design that allowed for contrast effects to be assessed within subjects. Two groups made choices about delayed or probabilistic rewards. After completing question blocks in which the amount was $\$ 5,000$ or $\$ 50$, subjects responded to questions with an intermediate amount $(\$ 475 / \$ 525)$. For Group Delay, the present value of the intermediate reward was greater after the $\$ 50$ block than after the $\$ 5,000$ block, whereas the opposite was obtained for Group Probability. The results from both experiments confirmed the predictions of reward contrast and suggested that the subjective value of a monetary reward varies inversely with the prior reward amount.
\end{abstract}

Two factors that influence decision making are delay and risk. For example, suppose that a lottery winner has to decide between a cash prize of $\$ 450$ available now or $\$ 500$ to be received in 1 year. Although the delayed reward is greater, he/she might choose the $\$ 450$, which could be spent right away. Thus, the subjective value of the $\$ 500$ at the time of choice is decreased by having to wait a year to receive it. The process whereby the value of an outcome decreases as a function of its delay is called temporal discounting or delay discounting (Chapman, 1998; Rachlin, Raineri, \& Cross, 1991).

Another factor that can influence decision making is risk - the degree of uncertainty that a reward might not be received (Kahneman \& Tversky, 1979; Tversky \& Kahneman, 1992). For example, consider a choice between receiving $\$ 450$ for sure or a $90 \%$ chance to receive $\$ 500$. Although the expected values of the choices are equal $(.90 \times 500=450)$, many might prefer $\$ 450$ for sure; at the time of choice, the subjective value of the $\$ 500$ may be lower because its receipt is uncertain. The decrease in value of an outcome due to its uncertainty is termed probability discounting (Green, Myerson, \& Ostaszewski, 1999; Rachlin et al., 1991). Delay and probability discounting are processes that influence behavior that could be described more broadly as intertemporal and risky choice, respectively (Myerson, Green, Hanson, Holt, \& Estle, 2003).

Research on intertemporal and risky choice has been informed by comparable studies with nonhumans. For example, the hyperbolic function proposed by Mazur (1984) to describe pigeons' choice between immediate and delayed food rewards provides a better account of humans' intertemporal choice than does the normative model of economics, which assumes an exponential discount function (Kirby, 1997; Myerson \& Green, 1995). In the domain of risky choice, Pietras, Locey, and Hackenberg (2003) showed that humans' choices between certain and uncertain alternatives varied with overall energy budget according to predictions of risk-sensitive models for foraging in behavioral ecology (Bateson \& Kacelnik, 1998; Caraco, Martindale, \& Whittam, 1980). Comparative research has documented similarities in temporal and probability discounting in both humans and nonhumans (e.g., Rodriguez \& Logue, 1988). In particular, hyperbolic functions provide a good description of results from experiments on both intertemporal and risky choice (with probability scaled as odds against in the latter; see Green \& Myerson, 2004, for a review).

One issue that has not been addressed in delay and probability discounting is the possible role of reward contrast effects (CEs). Typically, CEs are said to occur when the value or effectiveness of a stimulus varies inversely with the surrounding context. For example, Reynolds (1961) found that pigeons' rate of keypecking maintained by a constant variable-interval schedule increased or decreased, respectively, when the reinforcement rate provided by an alternative schedule was changed to extinction or a richer schedule (see Williams, 1983, 2002, for reviews). Such contrast effects are ubiquitous phenomena in research on conditioning and learning in nonhumans (Flaherty, 1996; Williams, 2002), as well as in studies of psychophysical judgment with humans (Lockhead, 2004). Applied to typical choice scenar-

R. C. Grace, randolph.grace@canterbury.ac.nz 
ios studied in temporal and probability discounting, which involve hypothetical monetary outcomes, reward contrast would predict that the subjective value of, say, a $\$ 500$ reward would seem subjectively larger or smaller, respectively, if the individual had recently made a decision about a smaller (e.g., \$50) or a larger (e.g., \$5,000) reward.

To the extent that contrast influences the subjective value of a reward, such effects could have strong effects on intertemporal and risky choice, because discounting rates vary depending on reward magnitude. Research with humans has consistently found that rates of temporal discounting decrease as the amount of reward increases (Johnson \& Bickel, 2002). To illustrate, someone might be indifferent between receiving $\$ 400$ now or $\$ 500$ in 1 year yet strongly prefer $\$ 15,000$ in 1 year to $\$ 12,000$ now. Because the delayed amount is $25 \%$ larger than the immediate amount in both cases, the preference for the delayed amount in the latter choice suggests that the discounting rate for $\$ 15,000$ must be less than the corresponding rate for $\$ 500$. Evidence for a magnitude effect in temporal discounting has been obtained with both real and hypothetical monetary rewards (Johnson \& Bickel, 2002; Madden, Begotka, Raiff, \& Kastern, 2003) and taxicab, hair salon, and restaurant tips (Green, Myerson, \& Schneider, 2003), as well as with outcomes related to health (Chapman, 1996), career decisions (Schoenfelder \& Hantula, 2003), and personal relationships (Tayler, Arantes, \& Grace, in press). By contrast, no evidence for amount-dependent discounting has been found in research with nonhumans (e.g., Grace, 1999; Green, Myerson, Holt, Slevin, \& Estle, 2004; Richards, Mitchell, de Wit, \& Seiden, 1997).

Magnitude of reward has also been found to affect rates of probability discounting, but in the opposite direction. Humans discount larger rewards more steeply as a function of the odds than they do smaller rewards (Christensen, Parker, Silberberg, \& Hursh, 1998; Estle, Green, Myerson, \& Holt, 2006; Green et al., 1999). That is, people show a greater degree of risk aversion with larger amounts (i.e., higher rate of discounting depending on odds against receiving the reward). For example, someone who is indifferent between $\$ 100$ for sure and a $40 \%$ chance of receiving $\$ 300$ is likely to prefer receiving $\$ 1,000$ for sure over a $40 \%$ chance of receiving $\$ 3,000$. The only comparable study with nonhumans is Mazur (1988), which showed that fixed-delay indifference points obtained with rats in an adjusting-delay procedure for certain versus uncertain rewards did not change systematically with the overall number of food pellets. Thus, there is no evidence of a magnitude effect on probability discounting with nonhumans, although additional research is warranted.

The goal of the present study was to determine whether reward contrast might influence delay and probability discounting in humans. We hypothesized that the subjective value of a monetary outcome might appear larger or smaller, respectively, if an individual had previously been making choices involving smaller or larger amounts. If reward value is influenced by CEs, rates of temporal and probability discounting should change because of the magnitude effects noted above. For example, the temporal-discounting rate for a $\$ 500$ reward should be reduced if it follows a smaller reward (\$50) but increased if it follows a larger reward $(\$ 5,000)$. For probability discounting, the opposite effects would be expected: The rate of probability discounting for $\$ 500$ should increase if it follows a smaller reward $(\$ 50)$ but decrease if it follows a larger reward $(\$ 5,000)$.

We will describe two experiments in which both betweensubjects and within-subjects designs were used to test for reward contrast effects in delay and probability discounting. In Experiment 1, different groups of subjects made decisions about a medium-sized reward (\$500) after previously making decisions about either a smaller $(\$ 50)$ or a larger $(\$ 5,000)$ reward in delay- and probability-discounting tasks. Reward contrast was assessed indirectly through changes in the discounting rates. In Experiment 2, we used a within-subjects design and evaluated reward contrast more directly in terms of changes in the present value of a discounted \$500 reward.

As a secondary aim, we sought to evaluate predictions on the basis of the view that impulsivity might underlie individual differences in discounting. Impulsivity is a personality trait or behavioral propensity that involves difficulty with delaying gratification and a low degree of risk aversion and is believed to be a contributing factor in addictive behavioral disorders, such as substance abuse and problem gambling (Moeller, Barratt, Dougherty, Schmitz, \& Swann, 2001). According to this view, impulsive individuals should have high rates of temporal discounting and low rates of probability discounting. However, previous studies have generally failed to show negative correlations between temporal- and probability-discounting rates, which raises questions about whether such rates measure the same construct as impulsivity (Holt, Green, \& Myerson, 2003; Myerson et al., 2003).

It is interesting to note that one way to interpret the magnitude effect on delay and probability discounting is in terms of a tendency to limit impulsive choice. That is, when the amount is relatively large, rates of delay and probability discounting shift in the direction associated with less impulsive choice (i.e., delay-discounting rates decrease and probabilitydiscounting rates increase), whereas when the amount is relatively small, rates of delay and probability discounting shift toward more impulsive choice. Thus, in Experiment 2, we also examined whether two psychometric variables that are commonly used in studies of impulsivity, the Barratt Impulsiveness Scale 11 (BIS-11; Patton, Stanford, \& Barratt, 1995) and the South Oaks Gambling Screen (SOGS; Lesieur \& Blume, 1987) would be correlated with delay- and probability-discounting rates and reward contrast.

\section{EXPERIMENT 1}

In Experiment 1, a between-subjects design was used to test for reward CEs in temporal and probability discounting. Subjects responded to a series of questions about hypothetical monetary outcomes. In the temporaldiscounting conditions, one outcome was an amount of money available immediately, whereas the other outcome was a larger, standard amount that was available after a delay. In the probability-discounting conditions, one outcome was certain, whereas the other outcome was to be obtained with a specified probability. In both conditions, a bisection algorithm was used to determine the amount of 
money available immediately or for sure that was equal in value to the delayed or probabilistic outcome, respectively. All the subjects completed two blocks of temporal- and probability-discounting questions. The amount of the immediate or certain outcome in the first block was either $\$ 50$ or $\$ 5,000$ (for different groups), whereas the corresponding amount for the second block was always $\$ 500$. If reward CEs were present, the temporal-discounting rate for $\$ 500$ should be greater for Group \$5,000 than for Group \$50, whereas the probability-discounting rate for $\$ 500$ should be greater for Group $\$ 50$ than for Group $\$ 5,000$.

\section{Method}

Subjects. Thirty-two students were recruited from the University of Canterbury. There were 20 females and 12 males, and the average age was 21.84 years. All received a $\$ 10$ petrol voucher in return for their participation in the experiment.

Procedure. The subjects were tested in small groups, seated individually in front of a computer in a laboratory room. The subjects were told that they would be presented with a series of questions displayed on the computer, which involved hypothetical choices between different amounts of money. They were asked to answer those questions as realistically and accurately as they possibly could. In some cases, the choices were between amounts of money available either immediately or after a delay. In other cases, the choices were between amounts of money to be received for certain ( $100 \%$ chance) or with a specified probability $(<100 \%$ chance). For each choice, there were two alternatives, displayed on the left and right sides of the screen. The subjects indicated their choice by pressing the " $\mathrm{z}$ " key (left alternative) or the "/" key (right alternative). If, at any time, an "incorrect" choice were made (i.e., the wrong key was pressed), the subjects could press the space bar to repeat the question. The selected alternative was highlighted in yellow for $0.75 \mathrm{sec}$ to provide feedback for the response. The computer program was written in FreePascal (www.freepascal.org).

The experiment comprised two pairs of trial blocks, one that involved delay discounting and one that involved probability discounting. In each block, indifference points were determined that were estimated to be equal in value to a standard amount. The standard amount for the second block in each pair was always $\$ 500$, and the subjects were randomly assigned to two groups in which the standard amount for the first block in each pair was either $\$ 50$ or $\$ 5,000$. The currency was New Zealand dollars, which at the time the experiment was conducted (April-May 2006) had an exchange rate of approximately NZ $\$ 1=$ US $\$ 0.63$.

In each block, the standard amount was studied at six different delays or probabilities. For the delay-discounting blocks, the delays were 1 month, 6 months, 1 year, 3 years, 5 years, and 10 years. For the probability-discounting blocks, the probabilities were $95 \%, 90 \%$, $70 \%, 40 \%, 10 \%$, and $5 \%$ (these were the same delays and probabilities used by Green et al., 1999). For each delay or probability, a series of questions was presented to determine an indifference point - the amount available immediately or for sure, respectively, that was equal in value to the delayed or probabilistic amount. To estimate indifference points efficiently, the amount of the immediate or certain option was changed across successive questions according to a bisection algorithm. For example, in the delay-discounting block with a standard amount of $\$ 500$ and a 1-year delay, the first question presented was $\$ 500$ delayed 1 year or $\$ 500$ now (i.e., the immediate amount was equal to the standard amount). If the immediate amount was chosen, the immediate amount for the next question would be the midpoint of the interval defined by the maximum and minimum potential values of the indifference point (which were defined at the start of each question series as the standard amount and $\$ 0$ ). The second question would then be $\$ 500$ delayed 1 year or $\$ 250$ now. If the delayed amount was now chosen, the immediate amount would be increased for the next question to $\$ 375$ (the midpoint of $\$ 250$ and $\$ 500$ ). If the delayed amount was chosen again, for the next question the immediate amount would be $\$ 437.50$ (the midpoint of $\$ 375$ and $\$ 500$ ). Thus, the immediate amounts converged on an indifference point over successive questions. For each question series, the indifference point was estimated as the immediate amount obtained after eight questions, which ensured that the estimates were precise (there were $2^{7}=128$ possible indifference points that were less than the delayed amount). Across the series, the change in the immediate amount based on the response to each question was halved each time and, thus, given a delayed amount of $\$ 500$, was $\$ 250, \$ 125, \$ 62.50, \$ 31.25, \$ 15.63, \$ 7.81, \$ 3.91$, and $\$ 1.95$ for the eight questions. A similar procedure was used to obtain indifference points in the probability-discounting blocks.

For each response in a series, there was a 1-sec interval after the 0.75 -sec feedback had been provided before the next question was presented. There was an interval of $3 \mathrm{sec}$ between question series within a block and an interval of $5 \mathrm{sec}$ between different blocks. As in Green et al. (1999), the delays and probabilities were presented in an increasing/decreasing order, respectively (so that discounting increased across trials within blocks for both delay and probability). Whether the delay-discounting blocks were completed first, followed by the probability-discounting blocks, or vice versa, was counterbalanced.

\section{Results}

Each indifference point was expressed as a present value $(P V)$ - that is, as a proportion of the standard reward in the question series. Figure 1 shows the average
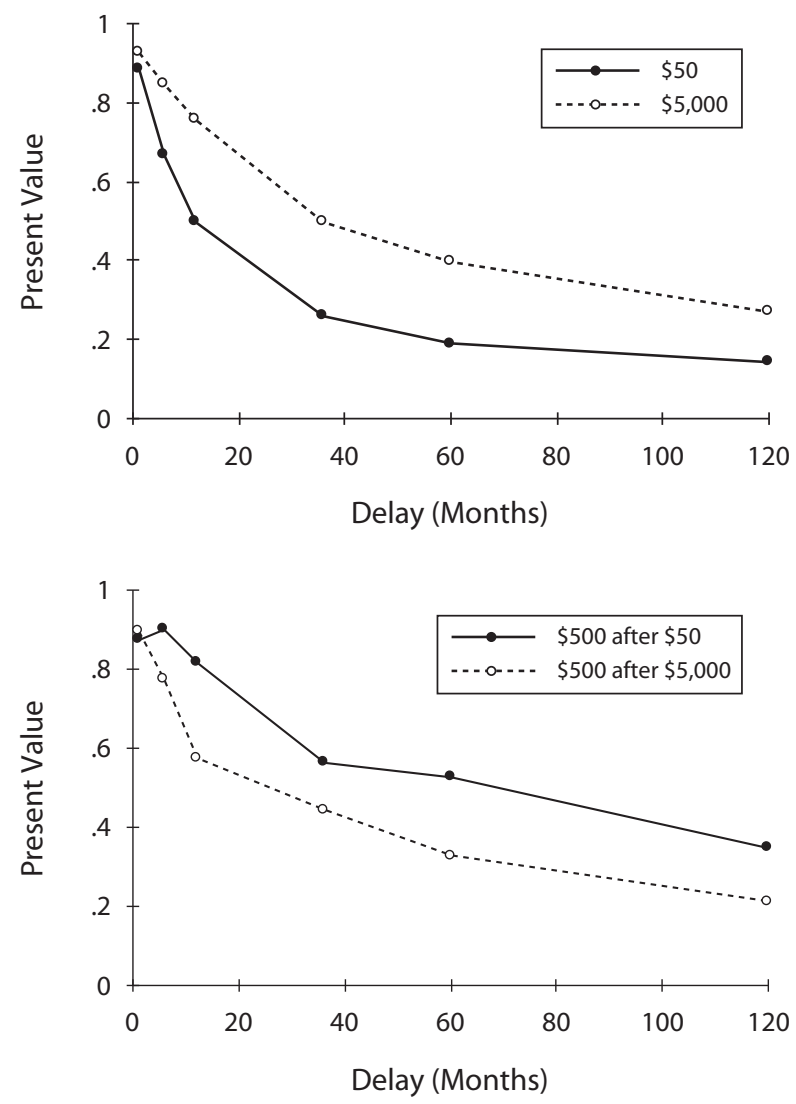

Figure 1. Average present value data for each question series from the delay-discounting task in Experiment 1. The upper panel shows results from the first block, in which the amount was either $\$ 50$ or $\$ \mathbf{5 , 0 0 0}$ for different groups of subjects. The lower panel shows results from the second block, in which the amount was $\$ \mathbf{5 0 0}$ for both groups. 


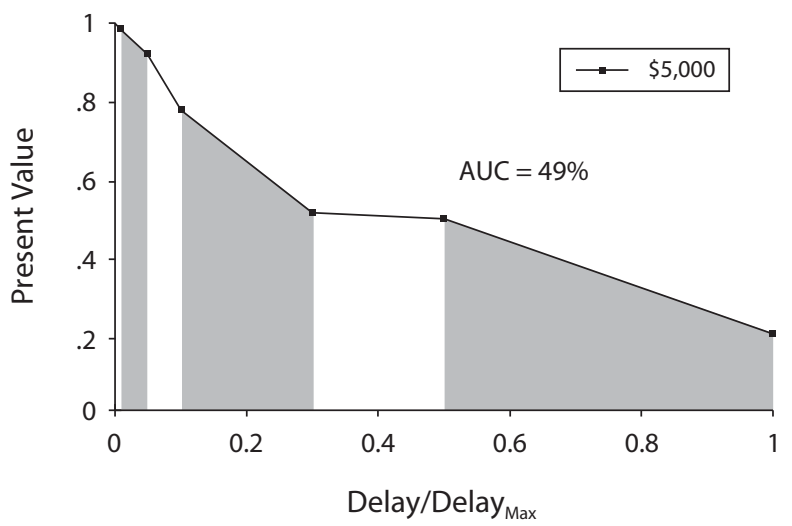

Figure 2. Illustration of how the area under the discounting curve (AUC) is calculated. Data are taken from a randomly selected subject in Experiment 1 for the delay-discounting task with $\$ 5,000$ as the standard amount. See the text for more explanation.

$P V$ for each question series in the delay-discounting task. Results for the first block ( $\$ 50$ or $\$ 5,000)$ are shown in the upper panel. As was expected, $P V$ decreased as delay increased for both amounts and was greater for $\$ 5,000$, suggesting that discounting rates varied inversely with amount, consistent with the magnitude effect. The results for the second block $(\$ 500)$ are shown in the lower panel. Overall, $P V$ for $\$ 500$ when $\$ 50$ had been the amount in the first block ( $\$ 500$ after $\$ 50)$ was greater than when $\$ 5,000$ had been the amount in the first block ( $\$ 500$ after $\$ 5,000)$. This is consistent with the predictions of reward contrast, because it suggests that the discounting rate was greater for $\$ 500$ after $\$ 5,000$, as required by the magnitude effect if $\$ 500$ was subjectively smaller when it followed the larger amount.

For a more precise assessment of delay-discounting rates, we calculated the area under the empirical discounting curve

\section{Delay Discounting}

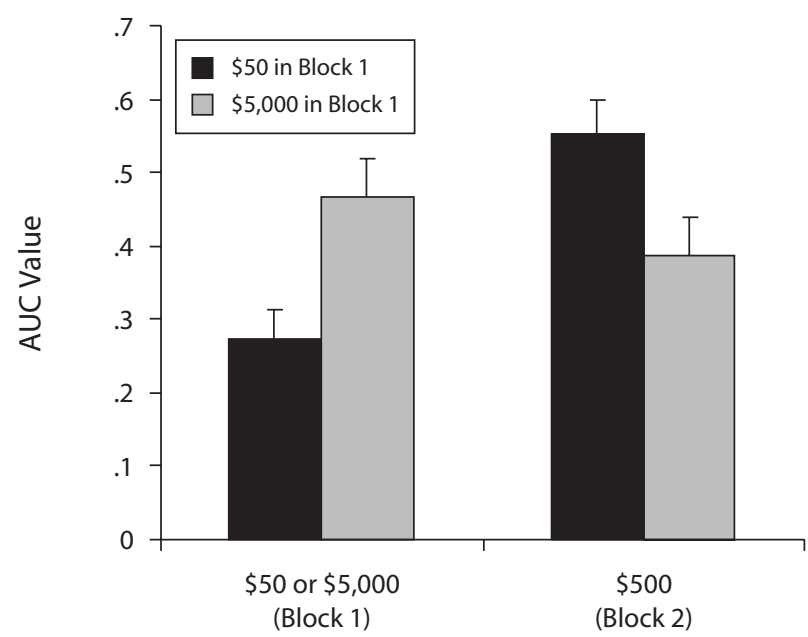

Figure 3. Average area under the discounting curve (AUC) values for the delay-discounting task in Experiment 1. Error bars indicate $\pm 1 S E$.
(AUC; Myerson, Green, \& Warusawitharana, 2001) for individual subjects. Figure 2 illustrates how the AUC is computed, using the data from a randomly selected subject for the $\$ 5,000$ block. $P V$ is plotted as a function of the normalized delay (i.e., delay divided by the maximum delay), and then areas for each trapezoidal segment, as indicated by the blank or shaded regions in Figure 2, are summed. The AUC has the advantage of not requiring a specific mathematical form for the discounting function and is an inverse measure of the discounting rate: Larger AUC values correspond to lower rates of discounting, and vice versa.

Figure 3 shows the average AUC values for the delaydiscounting data. As Figure 3 shows, discounting rates were greater for $\$ 50$ than for $\$ 5,000$ (mean $\mathrm{AUCs}=.27$ and .47 , respectively) and greater for $\$ 500$ after $\$ 5,000$ than for $\$ 500$ after $\$ 50$ (mean $\mathrm{AUCs}=.39$ and .55 ). A repeated measures ANOVA with first or second block of discounting questions as a within-subjects factor and amount during the first block ( $\$ 50$ or $\$ 5,000)$ as a between-groups factor confirmed these observations. There was a significant block $\times$ amount interaction $[F(1,30)=44.12, p<$ $.001]$, and planned comparisons showed that the AUC was greater for $\$ 5,000$ in the first block $[F(1,30)=8.94, p<$ $.01]$ and greater for $\$ 500$ after $\$ 50$ in the second block $[F(1,30)=5.80, p<.05]$. The main effect of block was significant $[F(1,30)=13.76, p<.001]$, indicating that AUC values were greater in the second block overall. The main effect of amount during the first block was not significant $[F(1,30)=0.06$, n.s. $]$. These results support the predictions of reward contrast: Delay-discounting rates for $\$ 500$ were greater (i.e., AUC values were lower) when it followed $\$ 5,000$ than when it followed $\$ 50$ and also confirmed that the expected effect of reward magnitude was obtained in the first block, with discounting rates greater for $\$ 50$ than for $\$ 5,000$.

Figure 4 shows the average $P V$ for each question series in the probability-discounting task. Overall, $P V$ was lower than in the delay task (cf. Figure 1), suggesting that the subjects discounted more rapidly as a function of odds against. $P V$ decreased as the odds against receiving the reward increased and was greater for $\$ 50$ than for $\$ 5,000$ (upper panel). This suggests that probability-discounting rates varied directly with amount, consistent with expectations based on the magnitude effect. Conversely, $P V$ was lower for $\$ 500$ after $\$ 50$ than for $\$ 500$ after $\$ 5,000$, suggesting that the subjective value of $\$ 500$ varied inversely with the amount in the first block.

A repeated measures ANOVA of the AUC data, shown in Figure 5, supported these conclusions. There was a significant interaction between block and amount during the first block $[F(1,30)=21.31, p<.001]$. A planned comparison between AUC values in the first block approached significance $[F(1,30)=2.96, p<.10]$, suggesting that AUC was greater for $\$ 50$ than for $\$ 5,000$, consistent with the expected effect of magnitude on probability discounting (i.e., a lower AUC for $\$ 5,000$ implies a higher discounting rate for the larger amount). A planned comparison for the data from the second block showed that AUC values were higher for $\$ 500$ when it followed $\$ 5,000$ than when it followed $\$ 50[F(1,30)=4.50, p<.05]$. This confirms 

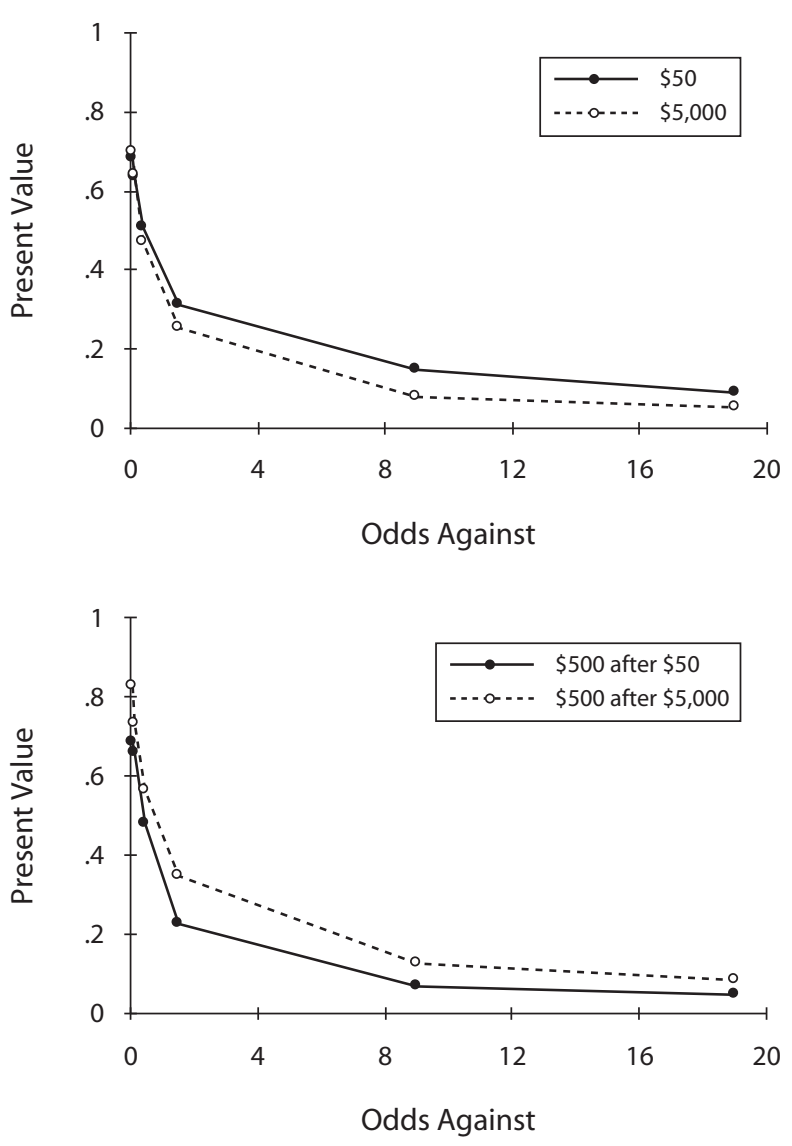

Figure 4. Average present value data for each question series from the probability-discounting task in Experiment 1. The upper panel shows results from the first block, in which the amount was either $\$ 50$ or $\$ \mathbf{5 , 0 0 0}$ for different groups of subjects. The lower panel shows results from the second block, in which the amount was $\$ \mathbf{5 0 0}$ for both groups.

\section{Probability Discounting}

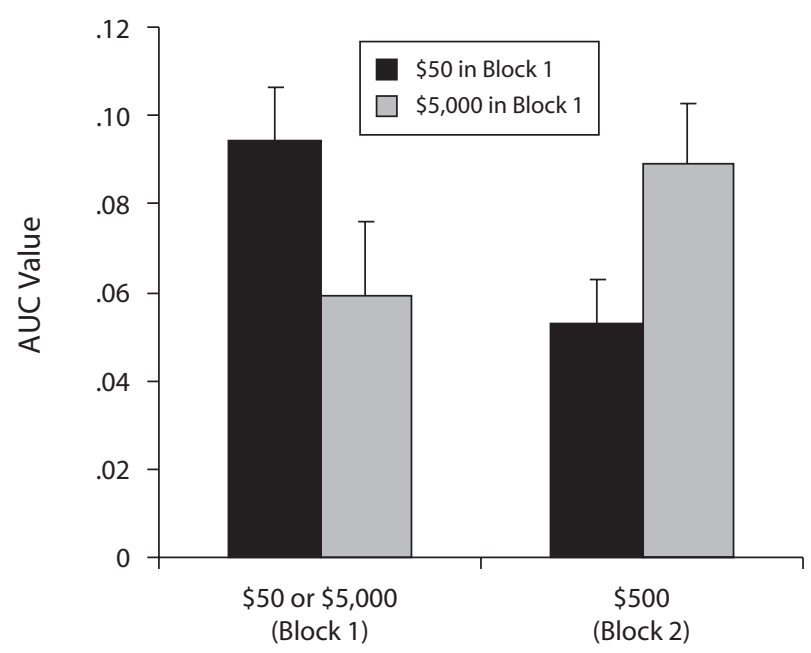

Figure 5. Average area under the discounting curve (AUC) values for the probability-discounting task in Experiment 1. Error bars indicate $\pm 1 S E$. the prediction of reward contrast, because it shows that discounting rates were higher (i.e., lower AUC) for $\$ 500$ when it followed $\$ 50$, suggesting that the subjective value of $\$ 500$ was larger when it followed the smaller amount.

To obtain an estimate of the size of the CE for delay and probability discounting, we calculated the average difference between the AUC values for the $\$ 500$ reward depending on whether the standard amount in the first block was $\$ 50$ or $\$ 5,000$, divided by the pooled standard deviation. The resulting values (Cohen's $d$ ) were 0.85 and -0.75 for delay and probability discounting, respectively (note that the signs were opposite because reward magnitude has opposite effects on delay and probability discounting).

Finally we examined the correlation between delay- and probability-discounting rates. For each subject, the average AUC value was computed for both delay and probability discounting. The correlation was positive $(r=.15)$ but failed to reach significance $(p>.40)$. Thus, there was no support for the hypotheses that individual differences in discounting can be linked to impulsivity as a personality trait, for which a negative correlation would be predicted.

\section{EXPERIMENT 2}

The results of Experiment 1 confirmed that reward CEs could be obtained for both delay and probability discounting. The discounting rate for the $\$ 500$ reward varied in the direction expected if its subjective value varied inversely with the standard amount in the first block, for both delay and probability discounting. To test the generality of CEs in Experiment 2, a different design was used in which CEs were assessed more directly, using a within-subjects design. Subjects responded to two series of discounting questions in which the standard amounts were $\$ 50$ and $\$ 5,000$. For Group Delay, indifference points for delays of 1 month, 6 months, 1 year, 3 years, 5 years, and 10 years were obtained for each series. For Group Probability, indifference points for reward probabilities of $95 \%, 90 \%$, $70 \%, 40 \%, 10 \%$, and $5 \%$ were obtained. After each series, an indifference point for an intermediate amount ( $\$ 475$ or $\$ 525$ ) was determined in a set of test questions. Different intermediate amounts for the two sets of test questions were used in order to make it less likely that the subjects would respond similarly in the two tests. If reward CEs are present, there should be an interaction between type of discounting (delay/probability) and standard amount $(\$ 50 / \$ 5,000)$ in the prior question series: The indifference point for the intermediate reward should be greater after $\$ 50$ than after $\$ 5,000$ for delay discounting but should be less after $\$ 50$ than after $\$ 5,000$ for probability discounting.

\section{Method}

Subjects. Sixty-four students were recruited from introductory psychology classes at the University of Canterbury. The subjects were randomly divided into Group Delay and Group Probability. For Group Delay $(n=32)$, there were 17 females and 15 males, with an average age of 22.41 years. For Group Probability, there 
were 14 females and 16 males, with an average age of 19.97 years. Two subjects assigned to Group Probability failed to complete the experiment, and their data were not included $(n=30)$. All received course credit in return for their participation.

Procedure. All details of the procedure were the same as those in Experiment 1, with the following exceptions. The subjects completed two series of discounting questions involving hypothetical amounts of money displayed on a computer screen, in which the standard amounts were $\$ 50$ and $\$ 5,000$. As in Experiment 1, the currency was New Zealand dollars, and at the time the study was conducted (MayJune 2007), the exchange rate was approximately NZ\$1 = US\$0.73. For Group Delay, indifference points were obtained for a range of delays ( 1 month, 6 months, 1 year, 3 years, 5 years, and 10 years), whereas for Group Probability, they were obtained for a range of reward probabilities $(95 \%, 90 \%, 70 \%, 40 \%, 10 \%$, and $5 \%)$. After each series, a set of test questions was conducted that determined an indifference point for an intermediate amount $(\$ 475$ or $\$ 525)$ for a 1 -year delay (Group Delay) or 70\% probability (Group Probability). After the first discounting series and intermediate amount, the subjects completed two paper-and-pencil questionnaires: the BIS-11 (Patton et al., 1995) and the SOGS (Lesieur \& Blume, 1987). Whether the standard amounts for the two series were $\$ 50$ and $\$ 5,000$ (or vice versa) and whether the intermediate amounts were $\$ 475$ and $\$ 525$ (or vice versa) were counterbalanced.

\section{Results}

As in Experiment 1, indifference points from the two discounting series were converted to $P V_{\text {s. }}$. Figure 6 shows
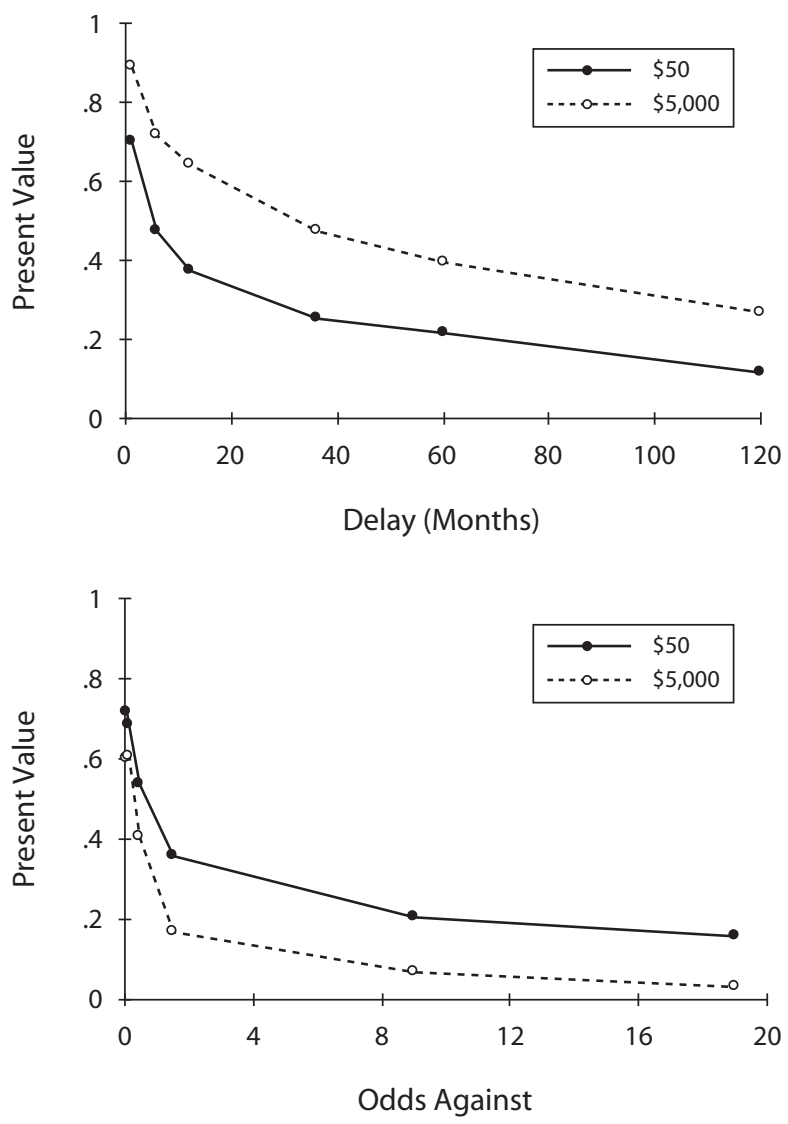

Figure 6. Average present value data for each question series for the delay-discounting (upper panel) and probability-discounting (lower panel) tasks in Experiment 2.

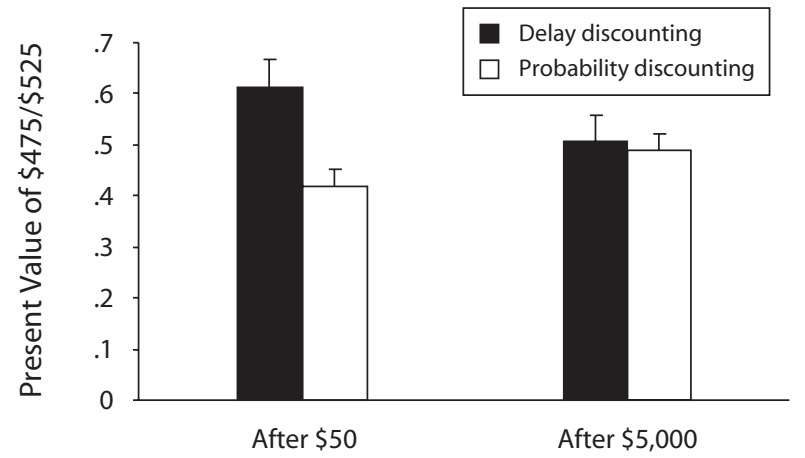

Figure 7. Results from the test series in Experiment 2. Shown are the average present values for the intermediate $(\$ 475 / \$ 525)$ amount discounted by 1-year delay or $70 \%$ probability of receipt, following a question block with either $\$ 50$ or $\$ 5,000$ as the standard amount. Results are shown separately for Group Delay (filled bars) and Group Probability (unfilled bars). Error bars indicate $\pm 1 S E$.

the average $P V$ for each question series for Group Delay (upper panel) and Group Probability (lower panel). For delay discounting, $P V$ was greater for $\$ 5,000$ than for $\$ 50$, whereas the opposite was obtained with probability discounting. Both these results are consistent with expectations based on the magnitude effect.

AUC values were computed for individual subjects; average values are shown in Figure 7. A repeated measures ANOVA with type of discounting as a between-subjects factor and amount $(\$ 50$ or $\$ 5,000)$ as a within-subjects factor showed a significant interaction $[F(1,60)=55.11$, $p<001]$. Planned comparisons showed that AUC was greater for $\$ 5,000$ than for $\$ 50$ for delay discounting $[F(1,60)=37.67, p<.001]$, whereas the opposite was true for probability discounting $[F(1,60)=19.28, p<$ $.001]$. This confirms that the expected effect of reward magnitude was obtained for both discounting tasks. The main effect of discounting type was also significant $[F(1,60)=19.52, p<.001]$, indicating that AUC values were overall greater (and discounting rates lower) for delay discounting.

To assess whether reward CEs were obtained, the indifference points for the intermediate amounts $(\$ 475 / \$ 525)$ from the test series were converted to $P V \mathrm{~s}$. A repeated measures ANOVA showed a significant interaction between amount and type of discounting $[F(1,60)=10.46$, $p<.01]$. As Figure 4 shows, this was a crossover interaction in which the $P V$ for the intermediate amount was greater after $\$ 50$ than after $\$ 5,000$ for delay discounting but was greater after $\$ 5,000$ than after $\$ 50$ for probability discounting. Planned comparisons showed that the difference in $P V$ for the intermediate amount was significant for delay discounting $[F(1,60)=7.97, p<.01]$ and approached significance for probability discounting $[F(1,60)=3.13, p=.08]$. This result is consistent with predictions based on reward contrast and suggests that the subjective value of the intermediate amount varied inversely depending on the standard amount in the previous question series. 
To obtain an estimate of the size of the CE within subjects, we calculated the difference between the $P V \mathrm{~s}$ of the intermediate amount following $\$ 5,000$ and $\$ 50$ (i.e., $P V_{\$ 475 / \$ 525 \text { after } \$ 5,000}-P V_{\$ 475 / \$ 525}$ after $\left.\$ 50\right)$. This yielded average CE estimates of $0.11(S E=.03)$ and $-0.07(S E=$ .05 ) for Group Delay and Group Probability, respectively. This suggests that the subjective value of the intermediate $(\$ 475 / \$ 525)$ reward changed by approximately $11 \%$ and $7 \%$, for Group Delay and Group Probability, respectively, depending on whether the subject had previously been making choices with a $\$ 5,000$ or a $\$ 50$ reward.

Finally, we examined correlations between the BIS-11 and SOGS, delay- and probability-discounting rates, and the CE estimates. The correlation between BIS-11 and SOGS was positive and significant $(r=.28, p<.05)$, consistent with the view that both measures are related to impulsivity. For Group Delay, correlations between the average AUC values and both BIS-11 and SOGS failed to reach significance ( $r \mathrm{~s}=-.23$ and -.23 , respectively), as did the correlations between CE and BIS-11/SOGS ( $r \mathrm{~s}=$ .17 and -.11 , respectively). For Group Probability, correlations between average AUC and BIS-11/SOGS were $r=$ -.18 and $r=.15$ (both n.s.), and between CE and BIS-11/ SOGS were $r=.11$ and $r=-.33$ (both n.s.). Thus, there was no evidence that BIS-11 and SOGS were correlated with measures of delay and probability discounting.

\section{DISCUSSION}

The goal of the present research was to determine whether reward CEs can influence humans' intertemporal and risky choices. Specifically, we asked whether the discounting rates and $P V \mathrm{~S}$ of delayed or probabilistic monetary rewards would change depending on whether subjects had previously been making choices about larger or smaller amounts of money. In Experiment 1, the subjects made choices about hypothetical $\$ 5,000$ or $\$ 50$ outcomes and then made choices about $\$ 500$ outcomes. Delay-discounting rates for the $\$ 500$ outcome were greater when the subjects had previously been making decisions about $\$ 5,000$, and probability-discounting rates for the $\$ 500$ outcome were greater when the subjects had previously been making decisions about $\$ 50$. Both these results are consistent with predictions of reward contrast and suggest that the subjective value of the $\$ 500$ reward varied inversely with the preceding amount. In Experiment 2, a design was used that allowed for CEs to be assessed more directly within subjects. Two groups made choices about delayed or probabilistic rewards. After completing blocks of questions in which the amount was $\$ 5,000$ and $\$ 50$, the subjects responded to a series of test questions with an intermediate amount ( $\$ 475 / \$ 525)$. For Group Delay, the $P V$ of the intermediate reward was greater after the $\$ 50$ block than after the $\$ 5,000$ block, whereas the opposite was obtained for Group Probability. This result is similar to that in Experiment 1 and suggests that the subjective value of the intermediate reward varied inversely with the amount in the prior question series.
Thus, our results confirm that CEs can play a role in humans' choice for delayed and probabilistic outcomes. The magnitude of the effects were fairly substantial: When assessed between subjects (Experiment 1), CE sizes (Cohen's $d$ ) for delay- and probability-discounting rates, as measured by AUC, were 0.85 and -0.75 , respectively. Experiment 2 permitted a direct calculation of the difference in the $P V$ of the $\$ 500$ reward: For delay discounting, the $P V$ changed by $11 \%$, depending on whether the amount in the prior question series was $\$ 5,000$ or $\$ 50$, and by $7 \%$ for probability discounting. Future research should examine whether the size of the CE depends on variables such as the relative amounts. For example, the subjective value of $\$ 500$ should decrease more in the context of previous choices about $\$ 50,000$ than in the context of those about $\$ 5,000$.

As was noted in the introduction, impulsivity offers a potentially attractive framework for understanding individual differences in discounting and the effect of reward magnitude: The opposite effects of magnitude on delay and probability discounting can both be understood in terms of an individual's choosing less impulsively when larger rewards are involved, in that he/she is more able to delay gratification (i.e., lower delay discounting rate) and more risk averse (i.e., higher probability-discounting rate). However, we found no evidence that responses on the BIS-11 and SOGS, two psychometric variables that are related to impulsivity, were correlated with delayor probability-discounting rates or with estimates of the CE.

Our study suggests that reward CEs in delay and probability discounting are a robust phenomenon, because they were obtained in two experiments with different designs. In Experiment 1, a between-subjects design was used in which contrast was assessed indirectly, through changes in discounting rates. In Experiment 2, a withinsubjects design was used in which contrast was measured more directly, in terms of changes in the $P V$ of a standard reward. It is important to note that contrast could be assessed in other ways. For example, after exposure to either a smaller or a larger reward, subjects could be asked about their willingness to purchase a good for an intermediate amount (e.g., an LCD TV for \$500) that previous research had shown that $50 \%$ of people would be willing to buy. Thus, future research should explore whether the generality of CEs extends beyond the kind of discounting task studied here.

Reward CEs may play an important role in real-world decision making. For example, a casino that has the croupier for a roulette table display a large stack of chips at the ready may increase the likelihood of risky or impulsive choice by gamblers, by making their bets seem relatively small in comparison. Legislators with budgetary responsibility who routinely make decisions involving billions of dollars may become more likely to agree to an urgent funding request in the millions. Investigating the influence of reward CEs in specific decision-making contexts and with more ecologically valid tasks (e.g., realistic gambling scenarios) should be a focus of future research. 
Although we tested only for CEs on reward amount, it is likely that other variables in the decision tasks, such as delay and probability, are also affected by contrast. This has methodological implications because it makes comparison of discounting rates across studies problematic: Discounting rates in a given study will likely depend on the range of reward magnitudes, delays, and probabilities.

Overall, the present study has demonstrated that the discounting rates in intertemporal and risky choice situations vary in the direction expected if the magnitude of a reward varies inversely with the reward context. Future research should examine whether individual differences in CEs might contribute to susceptibility to maladaptive choice - for example, problem gambling.

\section{AUTHOR NOTE}

These data were presented at the Annual Meeting of the Association for Behavior Analysis, May 2008 (Chicago). Address correspondence to R. C. Grace, Department of Psychology, University of Canterbury, Private Bag 4800, Christchurch, New Zealand (e-mail: randolph.grace@ canterbury.ac.nz).

\section{REFERENCES}

BAteson, M., \& KaCELnIK, A. (1998). Risk sensitive foraging: Decision making in variable environments. In R. Dukan (Ed.), Cognitive ecology: The evolutionary ecology of information processing and decision making (pp. 297-341). Chicago: University of Chicago Press.

Caraco, T., Martindale, S., \& Whittam, T. S. (1980). An empirical demonstration of risk-sensitive foraging preferences. Animal Behaviour, 28, 820-830.

Chapman, G. B. (1996). Expectations and preferences for sequences of health and money. Organizational Behavior \& Human Decision Processes, 67, 59-75.

Chapman, G. B. (1998). Sooner or later: The psychology of intertemporal choice. In D. Medin (Ed.), The psychology of learning and motivation (Vol. 38, pp. 83-113). San Diego: Academic Press.

Christensen, J., Parker, S., Silberberg, A., \& Hursh, S. (1998). Tradeoffs in choice between risk and delay depend on monetary amounts. Journal of the Experimental Analysis of Behavior, 69, 123-139.

Estle, S. J., Green, L., Myerson, J., \& Holt, D. D. (2006). Differential effects of amount on temporal and probability discounting of gains and losses. Memory \& Cognition, 34, 914-928.

FlaherTy, C. F. (1996). Incentive relativity. New York: Cambridge University Press.

GraCE, R. C. (1999). The matching law and amount-dependent exponential discounting as accounts of self-control choice. Journal of the Experimental Analysis of Behavior, 71, 27-44.

Green, L., \& Myerson, J. (2004). A discounting framework for choice with delayed and probabilistic rewards. Psychological Bulletin, 130, 769-779.

Green, L., Myerson, J., Holt, D., Slevin, R., \& Estle, J. (2004). Discounting of delayed food rewards in pigeons and rats: Is there a magnitude effect? Journal of the Experimental Analysis of Behavior, 81, 39-50.

Green, L., Myerson, J., \& Ostaszewski, P. (1999). Amount of reward has opposite effects on the discounting of delayed and probabilistic outcomes. Journal of Experimental Psychology: Learning, Memory, \& Cognition, 25, 418-427.

Green, L., Myerson, J., \& Schneider, R. (2003). Is there a magnitude effect in tipping? Psychonomic Bulletin \& Review, 10, 381-386.

Holt, D. D., Green, L., \& Myerson, J. (2003). Is discounting impulsive? Evidence from temporal and probability discounting in gambling and non-gambling college students. Behavioural Processes, 64, 355-367.
JoHnson, M. W., \& BicKEL, W. K. (2002). Within-subject comparison of real and hypothetical money rewards in delay discounting. Journal of the Experimental Analysis of Behavior, 77, 129-146.

Kahneman, D., \& Tversky, A. (1979). Prospect theory: An analysis of decision under risk. Econometrica, 47, 263-291.

KIRBY, K. N. (1997). Bidding on the future: Evidence against normative discounting of delayed rewards. Journal of Experimental Psychology: General, 126, 54-70.

Lesieur, H. R., \& Blume, S. B. (1987). The South Oaks Gambling Screen (The SOGS): A new instrument for the identification of pathological gamblers. American Journal of Psychiatry, 144, 1184-1188.

LOCKHEAD, G. R. (2004). Absolute judgments are relative: A reinterpretation of some psychophysical ideas. Review of General Psychology, 8, 265-272.

Madden, G. J., BegotKa, A. M., RaifF, B. R., \& Kastern, L. L. (2003). Delay discounting of real and hypothetical rewards. Experimental \& Clinical Psychopharmacology, 11, 139-145.

MAZUR, J. E. (1984). Tests of an equivalence rule for fixed and variable reinforcer delays. Journal of Experimental Psychology: Animal Behavior Processes, 10, 426-436.

MAZUR, J. E. (1988). Choice between small certain and large uncertain reinforcers. Animal Learning \& Behavior, 16, 199-205.

Moeller, F. G., Barratt, E. S., Dougherty, D. M., Schmitz, J. M., \& Swann, A. C. (2001). Psychiatric aspects of impulsivity. American Journal of Psychiatry, 158, 1783-1793.

Myerson, J., \& Green, L. (1995). Discounting of delayed rewards: Models of individual choice. Journal of the Experimental Analysis of Behavior, 64, 263-276.

Myerson, J., Green, L., Hanson, J. S., Holt, D. D., \& Estle, S. J. (2003). Discounting delayed and probabilistic rewards: Processes and traits. Journal of Economic Psychology, 24, 619-635.

Myerson, J., Green, L., \& Warusawitharana, J. (2001). Area under the curve as a measure of discounting. Journal of the Experimental Analysis of Behavior, 76, 235-243.

Patton, J. H., Stanford, M. S., \& Barratt, E. S. (1995). Factor structure of the Barratt impulsiveness scale. Journal of Clinical Psychology, 51, 768-774.

Pietras, C. J., Locey, M. L., \& Hackenberg, T. D. (2003). Human risky choice under temporal constraints: Tests of an energy-budget model. Journal of the Experimental Analysis of Behavior, 80, 59-75.

Rachlin, H., RaIneri, A., \& Cross, D. (1991). Subjective probability and delay. Journal of the Experimental Analysis of Behavior, 55, 233-244.

REYNOLDS, G. S. (1961). Behavioral contrast. Journal of the Experimental Analysis of Behavior, 4, 57-71.

Richards, J. B., Mitchell, S. H., De Wit, H., \& Seiden, L. S. (1997). Determination of discount functions in rats with an adjusting-amount procedure. Journal of the Experimental Analysis of Behavior, 67, 353-366.

Rodriguez, M. L., \& Logue, A. W. (1988). Adjusting delay to reinforcement: Comparing choice in pigeons and humans. Journal of Experimental Psychology: Animal Behavior Processes, 14, 105-117.

Schoenfelder, T. E., \& Hantula, D. A. (2003). A job with a future? Delay discounting, magnitude effects, and domain independence of utility for career decisions. Journal of Vocational Behavior, 62, 43-55.

Tayler, S., Arantes, J., \& Grace, R. C. (in press). Temporal discounting for monetary and close relationship outcomes. Personal Relationships.

TVersky, A., \& Kahneman, D. (1992). Cumulative prospect theory: An analysis of decision under uncertainty. Journal of Risk \& Uncertainty, 5, 297-323.

WiLliams, B. A. (1983). Another look at contrast in multiple schedules. Journal of the Experimental Analysis of Behavior, 39, 345-384.

Williams, B. A. (2002). Behavioral contrast redux. Animal Learning \& Behavior, 30, 1-20.

(Manuscript received January 29, 2009; revision accepted for publication February 5, 2009.) 